\title{
Measurement experiences with FluxSet digital D/I station
}

\author{
László Hegymegi ${ }^{1}$, János Szöllôsy ${ }^{2}$, Csaba Hegymegi ${ }^{1}$, and Ádám Domján ${ }^{1}$ \\ ${ }^{1}$ MinGeo Ltd., Budapest, Hungary \\ ${ }^{2}$ Independent contractor \\ Correspondence to: László Hegymegi (hegymegi@mingeo.com)
}

Received: 3 March 2017 - Discussion started: 6 March 2017

Revised: 30 May 2017 - Accepted: 30 May 2017 - Published: 25 July 2017

\begin{abstract}
Geomagnetic observatories use classical theodolites equipped with single-axis flux-gate magnetometers known as declination-inclination magnetometers (DIM) to determine absolute values of declination and inclination angles. This instrument and the measurement method are very reliable but need a lot of handwork and experience. The authors developed and built a non-magnetic theodolite which gives all measurement data in digital form. Use of this instrument significantly decreases the possibility of observation errors and minimises handwork. The new instrument is presented in this paper together with first measurement results in comparison to the classical DIM.
\end{abstract}

\section{Introduction}

Regular absolute measurement of the geomagnetic field using classical DIM is required to ensure the stability and calibration of geomagnetic observatory data (St-Louis et al., 2012). A digital non-magnetic DIM known as the declination/inclination Digital Station 1 (DS-1) was designed and built to improve the quality and reliability of the manual process.

This instrument measures declination and inclination angles of the geomagnetic field vector with digital angle encoders as well as the magnetic field measured by the attached single-axis FluxSet magnetometer. Data are transmitted via radio to a central processor unit. This unit also receives time stamp information from a built-in GPS receiver. All these data are stored in the central unit for later processing.

The central processor unit has a serial port which connects to a local-scalar absolute magnetometer. This solution can be useful in case of field measurements when the distance to the nearest observatory is too great to provide adequate correlation.

Data visualisation is made by portable devices such as laptops, tablets or e-book readers using Wi-Fi communication with the $802.11 \mathrm{AC}$ standard. The connected unit is also used to set up configuration parameters. At the end of the measurements all data can be exported to an xls or csv formatted table.

All the instrumentation run on built-in batteries for at least $3 \mathrm{~h}$ and can be charged using $12 \mathrm{~V} \mathrm{DC}$ or $230 \mathrm{~V} \mathrm{AC}$. The data visualisation display shows the power state of the batteries for every component of the DS-1.

Comparative measurements were made between the DS1 and the Nagycenk Observatory's absolute instrument on 19 December 2016. The final appraisal shows that the DS-1 is suitable for absolute control measurements, and it is more convenient, user friendly and effective than the traditional DIM. More comparison measurements will be performed for much longer periods with weekly intervals to check the operation stability and precision of the DS-1.

\section{Hardware construction}

Hardware components of the first model of the DS-1 equipment is shown on Fig. 1. It has three main parts: the theodolite with a single-axis magnetometer, the central processor and the display unit.

The most important part is the modified Zeiss THEO 020 theodolite mechanics, with built-in digital encoders and a FluxSet magnetometer mounted on its telescope. All steel and all inner optical parts of the original theodolite are removed and original graduated circles are replaced with the new glass circles of the digital angle encoder. The vertical encoder chip is fixed on the pendulum of the theodolite to 
Declinationlinclination Digital Station 1

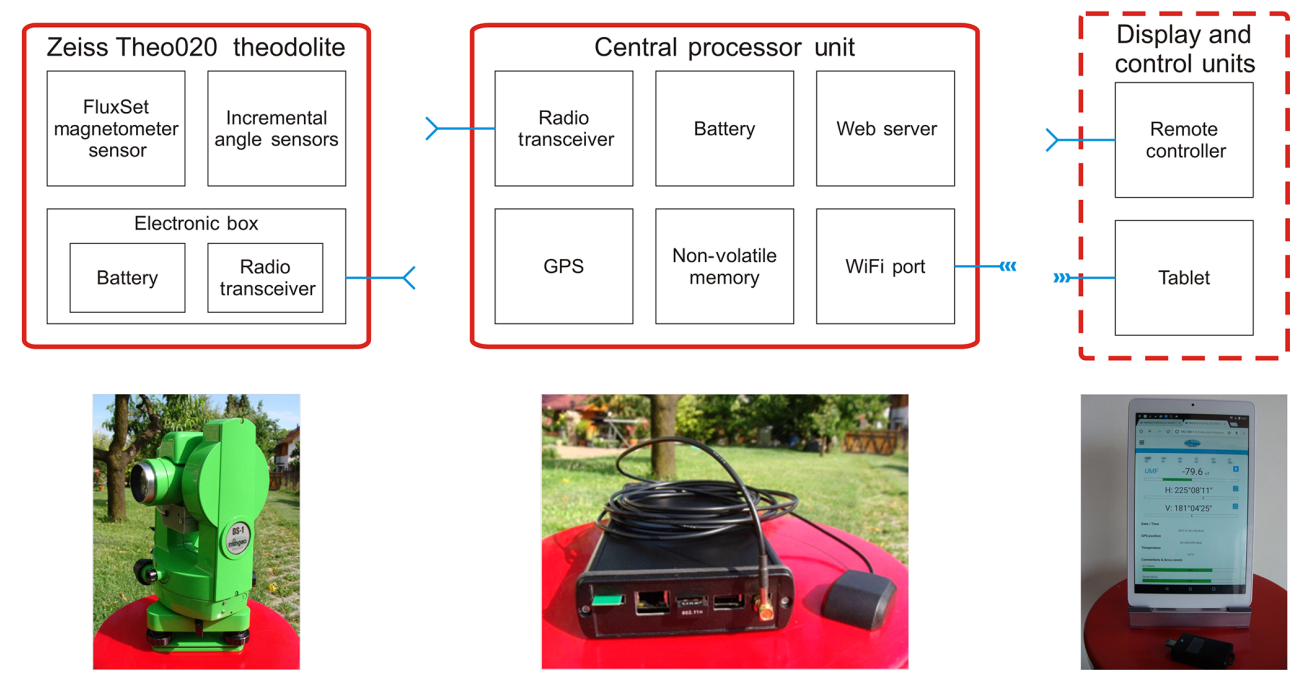

Figure 1. Declination/inclination Digital Station 1 (DS-1).

eliminate reading differences caused by small levelling errors. This modification has not any influence on the smooth operation of the pendulum, which was proved experimentally.

The DS-1 instrument is built with a FluxSet magnetometer. Its operating principle is similar to the pulse-position type fluxgate magnetometer. The practical advantage of these magnetometers is that their signal can be easily converted into a binary signal and the measurement of magnetic field is reduced to a high-precision time measurement through the displacement of the magnetisation curve produced by the external field. The magnetometer measures the axial magnetic field at the probe. The transverse sensitivity is negligible. The probe of the device is a small coil around a high-permeability (1 mm wide) amorphous metal strip (Vértessy et al., 2000).

The magnetic sensor was placed on the top of the telescope. The magnetometer has a resolution of $0.1 \mathrm{nT}$ and the dynamic range of operation is $\pm 3000 \mathrm{nT}$. In this range the magnetometer is linear as shown on Fig. 2.

All the electronics of the angle encoders and magnetometer are built into the electronics housing and fixed on the bottom of the telescope of the instrument. It also contains the battery with a charge controller and a radio unit to provide cableless connection to the central processor and to the remote controller. Owing to this design, the magnetic effect of the printed circuit board and battery in the electronic box is cancelled by measurements taken in four positions, because it rotates with the telescope (Gilbert and Rasson, 1998). We suppose that the box has not any induced magnetism; however it was not tested yet.

The central processor unit is responsible for the Wi-Fi network deployment, data storage and radio communication between the hardware components, and provides a web server with a GUI web page. The central processor unit receives measured magnetic and angle values and measurement commands sent by the observer via remote controller. It has a built-in GPS receiver to supply time stamps for measurement data and geographical coordinates which can be useful for field use. It can be connected to the absolute scalar magnetometer if these data are not available from another external source. The unit has its own battery, ensuring operation for about $6 \mathrm{~h}$. It has some steel parts; therefore its magnetic effects from 1 and $2 \mathrm{~m}$ are 3.0 and $0.3 \mathrm{nT}$, respectively. It must be placed at least $2 \mathrm{~m}$ away from the absolute pillar. The central processor unit electronics does not have a builtin display; it only has LEDs indicating regular operation and charging process.

The display function is performed by a tablet equipped with Wi-Fi. A large-screen tablet is included in the package of DS-1 equipment, the magnetic effects of which are 3.5 and $0.5 \mathrm{nT}$ from 1 and $2 \mathrm{~m}$. Instead of this tablet, the user can use any Wi-Fi-capable device which has a web browser. After DS-1 installation the operator can follow the absolute magnetic measurement by watching the GUI web page on the tablet and saving the measurements via the remote controller.

\section{Measurement experience and results}

The measurement procedure with DS- 1 is the same as with DIM. It should be used like a classical DIM. On the display screen in addition to magnetometer output, horizontal $(H)$ and vertical $(V)$ angle values with some other information is shown on the web server display. There is also a reminder of the following sensor and telescope position in the measurement protocol (Fig. 3). When a button is pushed on the remote controller, the actual data are stored in the memory of DS-1. 

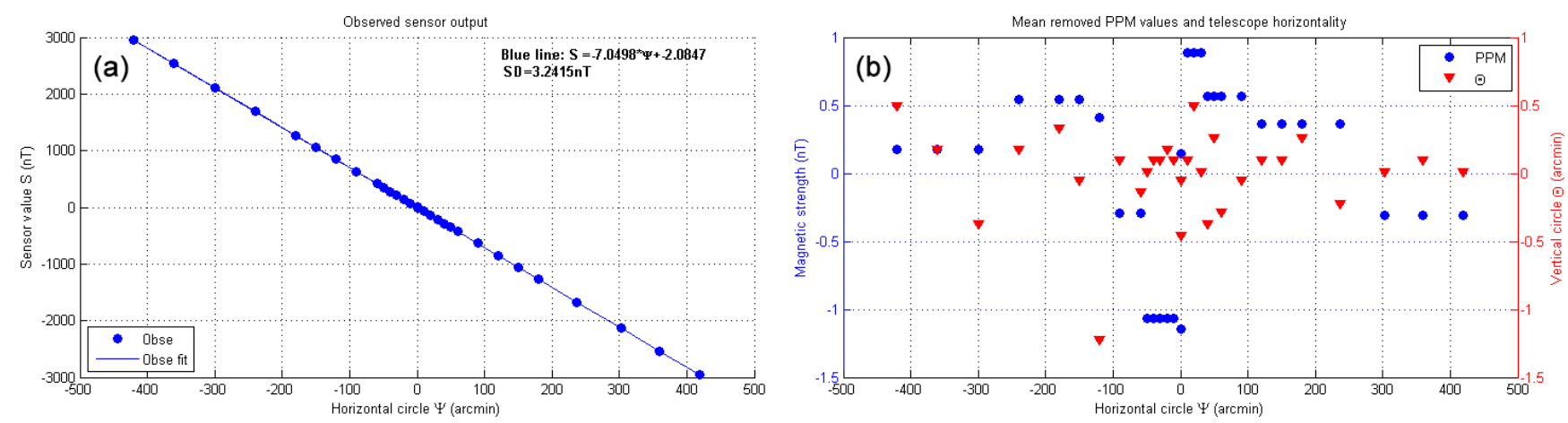

Figure 2. FluxSet magnetometer is linear inside the $\pm 3000 \mathrm{nT}$ range and the observations deviate with $3.2 \mathrm{nT}$ from the calculated line (a), while the scalar (PPM) readings changed by a maximum of $2 \mathrm{nT}$ during the 10 min linearity check test (b).

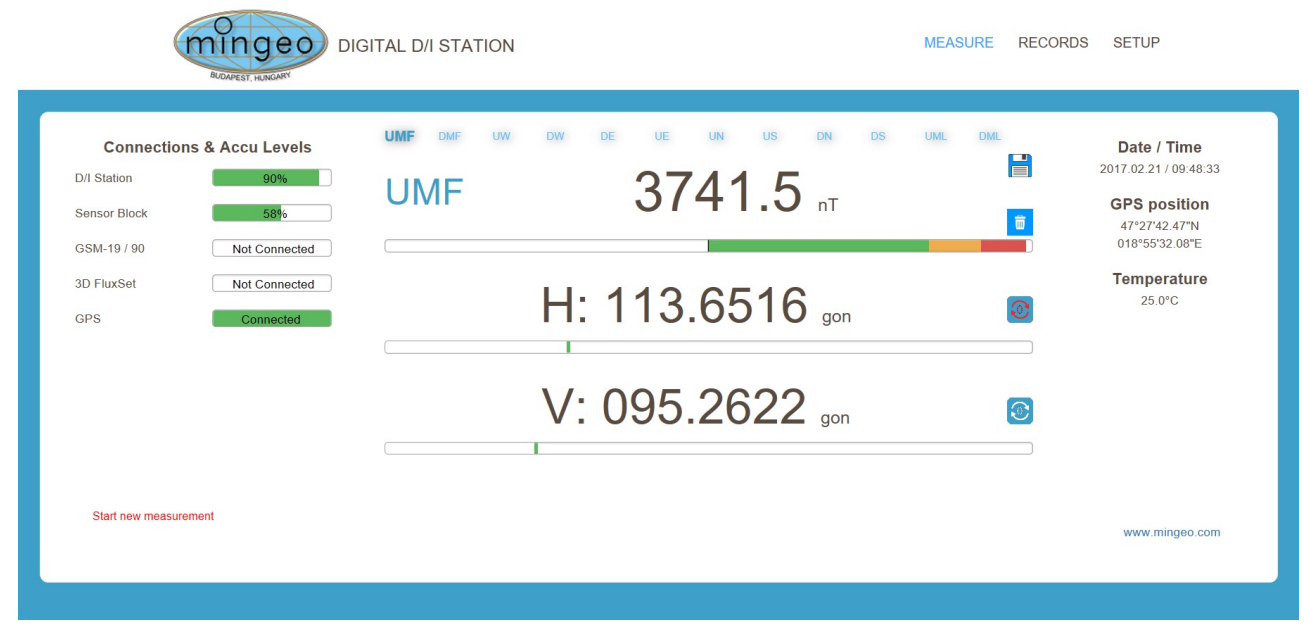

Figure 3. The measurement tab of the GUI web page. On the left side is connection and battery life information. On the right side is date, time, GPS and temperature data. In the middle of the tab the current magnetic sensor reading is shown with its corresponding horizontal and vertical angle values. In the top and middle panels the predefined measurement sequence can be seen, which begins with the first azimuth reading with the telescope in an upward position (UMF) and emphasis on the ongoing position.

In order to judge the quality of absolute values provided by DS-1 equipment a 1 day-long instrument comparison test was carried out at Nagycenk Observatory (NCK) on 19 December 2016. The reference instrument was NCK's main absolute instrument, a ZEISS THE 010B equipped with DMI G type fluxgate magnetometer. The measurements were taken alternately by two operators.

During the observations the geomagnetic activity was quiet. The difference between maximum and minimum value was within $21 \mathrm{nT}$ at $X$ and $Y$ horizontal components (Clarke et al., 2013). At the NCK measurement procedures the duplicate, symmetrical scheme (MDIIDM, $M$ stands for the azimuth (mark), $D$ is the declination angle, $I$ is the inclination angle) is used to produce a set of readings with the reference instrument in order to easily identify any error. Typical operators require about 20-25 min to complete this schema with both instruments. However, with the DS- 1 the simplified scheme (MDIM) was used similarly to the 2016
IAGA Observatory Workshop in Dourbes. The use of different schemas made the analysis more complicated, but it had no effect on the results.

During the comparison test on 19 December 2016 we completed 15 series with the DS- 1 and 3 series with the reference instrument using the simplified and the duplicate scheme, respectively. The instruments were installed on the same absolute pillar of the NCK observatory. Absolute measurements with the reference instrument were at the middle and the end of the day. Results are shown on Fig. 4a and b. For comparison $D, I$ angles are also calculated from the $1 \mathrm{~min}$ average of variometer data of NCK, THY and WIC observatories (Zhang et al., 2016). At NCK the registration of geomagnetic field variations is carried out by two sets of triaxial fluxgate magnetometers, namely by the ARGOS and the DRXX systems (Ádám et al., 2009). The variometer data from the other two observatories were downloaded from the INTERMAG- 

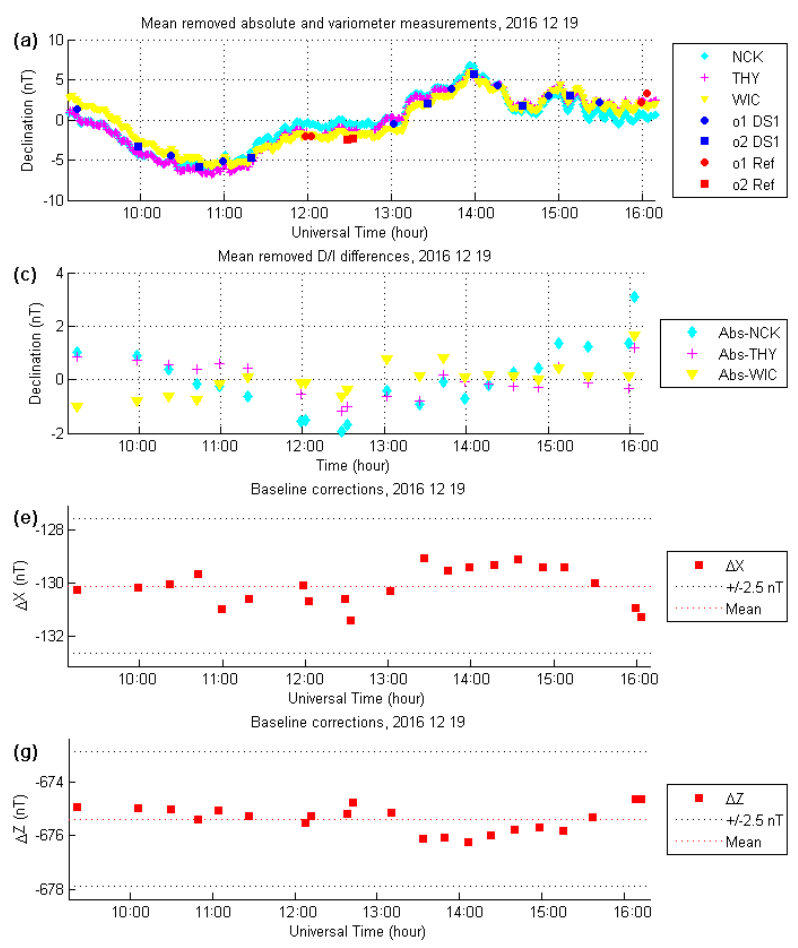
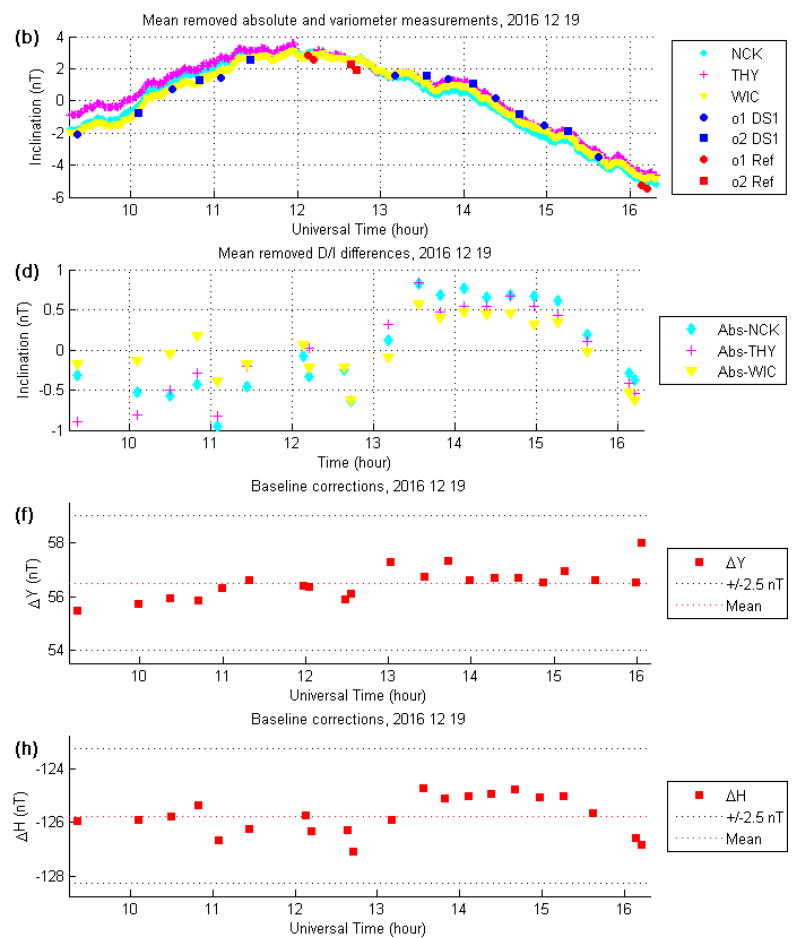

Figure 4. (a-b) The mean-removed declination and inclination values in nT unit. The circle and square marks refer to observations 1 and 2 , respectively. The red colour corresponds to the absolute measurements with the reference instrument, while the blue corresponds to DS-1 equipment. The cyan diamond, magenta cross and the yellow triangles designate the calculated declination and inclination from the 1 min variometer data of the NCK, THY and WIC observatories, respectively. (c-d) Difference between the absolute and averaged $D, I$ angles. (e-h) The baseline corrections calculated from absolute data for the related components. The correction values accomplished the adopted confidence limit in the INTERMAGNET specification (definitive baseline data accuracy: $\pm 5 \mathrm{nT}$ ) (St-Louis et al., 2012).

NET website. It is clearly seen that the absolute $D, I$ angles follow the calculated 1 min variometer data during the day.

On Fig. $4 \mathrm{c}$ and d we show the difference between measured $D, I$ angles and average $D, I$ values computed from variometer data of NCK, THY and WIC observatories for the period of measurements. Statistical results are shown on Table 1. The standard deviation of the differences is below $1.5 \mathrm{nT}$ for every component.

We calculated $\Delta X, \Delta Y, \Delta Z, \Delta H$ corrections which have to be added to $X, Y, Z$ and $H$ baseline components, according to Sect. 5.1 of Jankowski and Sucksdorff (1996, p. 86) to serve as a secondary quality check. For these calculations we used the raw readings of the continuously operating proton precession scalar magnetometer and variometer of the NCK observatory. The corresponding plots of the corrections shown on the Fig. 4e-h. Statistical properties are summarised in Table 2. All corrections show low standard deviation. They are within the $\pm 2.5 \mathrm{nT}$ interval, which suggests that it could be a good source for baseline fitting. Furthermore, these standard deviations demonstrate that the DS- 1 is suitable for supplying high-quality data for IMOs.

Certainly 1 day data are not enough for an accurate baseline determination, but it is a good sign that the data exhibit low-scatter characteristics. Our next mission is to perform
Table 1. The statistical parameters of the differences between absolute and variometer declination/inclination angles.

\begin{tabular}{lrrr}
\hline \multicolumn{4}{c}{ Declination differences } \\
\hline & Abs-NCK (nT) & Abs-THY (nT) & Abs-WIC (nT) \\
\hline Mean & -73.66 & -52.68 & 66.16 \\
SD & 1.23 & 0.64 & 0.61 \\
Variance & 1.52 & 0.41 & 0.37 \\
Max-min & 5.02 & 2.33 & 2.68 \\
\hline \multicolumn{4}{c}{ Inclination differences } \\
\hline Mean & Abs-NCK (nT) & Abs-THY (nT) & Abs-WIC (nT) \\
SD & 392.91 & -207.46 & 79.31 \\
Variance & 0.56 & 0.57 & 0.37 \\
Max-min & 0.32 & 0.33 & 0.14 \\
& 1.78 & 1.74 & 1.19 \\
\hline
\end{tabular}

at least a week-long observation with DS-1 equipment to see the data quality of this instrument and check whether it meets the INTERMAGNET standard (St-Louis et al., 2012; Newitt et al., 1996). 
Table 2. Statistics of baseline corrections at all and individual instruments.

\begin{tabular}{lrrrr}
\hline \multicolumn{5}{c}{ Statistics of all absolute measurements } \\
\hline & $\Delta X(\mathrm{nT})$ & $\Delta Y(\mathrm{nT})$ & $\Delta Z(\mathrm{nT})$ & $\Delta H(\mathrm{nT})$ \\
\hline Mean & -130.11 & 56.52 & -675.37 & -125.76 \\
SD & 0.71 & 0.59 & 0.49 & 0.71 \\
Variance & 0.50 & 0.35 & 0.24 & 0.51 \\
Max-min & 2.32 & 2.57 & 1.64 & 2.37 \\
\hline
\end{tabular}

Statistics of DS-1 equipment absolute measurements

\begin{tabular}{lrrrr}
\hline & $\Delta X(\mathrm{nT})$ & $\Delta Y(\mathrm{nT})$ & $\Delta Z(\mathrm{nT})$ & $\Delta H(\mathrm{nT})$ \\
\hline Mean & -129.82 & 56.50 & -675.52 & -125.47 \\
SD & 0.57 & 0.55 & 0.46 & 0.58 \\
Variance & 0.32 & 0.30 & 0.21 & 0.34 \\
Max-min & 1.88 & 1.88 & 1.34 & 1.93 \\
\hline
\end{tabular}

Statistics of ref. instrument absolute measurements

\begin{tabular}{lrrrr}
\hline & $\Delta X(\mathrm{nT})$ & $\Delta Y(\mathrm{nT})$ & $\Delta Z(\mathrm{nT})$ & $\Delta H(\mathrm{nT})$ \\
\hline Mean & -130.83 & 56.56 & -675.00 & -126.47 \\
SD & 0.49 & 0.76 & 0.38 & 0.47 \\
Variance & 0.24 & 0.57 & 0.15 & 0.22 \\
Max-min & 1.34 & 2.13 & 0.91 & 1.36 \\
\hline
\end{tabular}

\section{Conclusions}

The DS-1 equipment is good enough to determine the absolute values of $D, I$ elements of the Earth's magnetic field vector based on statistics of the one full day of absolute data measured by the Digital Station 1. The confidence limit of calculated baseline corrections are verified to meet the INTERMAGNET standards and specifications (St-Louis et al., 2012; Newitt et al., 1996).

Use of DS-1 equipment is very convenient, especially at polar or equatorial regions, where a classical instrument must use a $90^{\circ}$ eyepiece to read the vertical circle. Another benefit of the DS-1 equipment is that most, if not all, transcription errors are eliminated. This is especially useful in the field or when the operator uses the "residual" method.

The DS-1 equipment can be employed for the repeat station surveys. A local-scalar magnetometer can be connected to the serial port of the central processor unit giving an opportunity to produce absolute elements of the geomagnetic field vector.

\section{Future work}

We are planning to carry out a 1-year experiment in cooperation with the NCK observatory's staff to compare DS-1 equipment to the NCK observatory's main instrument in production of annual baseline.

We also plan to check the instrument according to the ISO 17123-3 standard (ISO 17123-3:2001, 2001).
Due to the convenient design and simple data acquisition with DS-1, it opens the possibility to test and evaluate new and more sophisticated absolute measurement routines beyond the null or residual methods. This equipment enables the observer to obtain much more data in a shorter time. A new method is currently under development, such as at the Niemegk observatory (H.-P. Brunke and J. Matzka, personal communication, 2016; Brunke and Matzka, 2017) which can be tested easily with DS-1. Common experiments are planned.

Data availability. The raw absolute magnetic measurement, variometer and scalar data measured at NCK are in the Supplement. The corresponding THY, WIC variometer data can be downloaded from the INTERMAGNET website (http://www.intermagnet.org/ data-donnee/download-eng.php).

\section{The Supplement related to this article is available online at https://doi.org/10.5194/gi-6-279-2017-supplement.}

Competing interests. The authors declare that they have no conflict of interest.

Acknowledgements. We are thankful to the staff of Geodetic and Geophysical Institute of Hungary (Judit Szendrői, Viktor Wesztergom) who provided expertise which greatly assisted the research, and the possibility to compare our instrument with theirs and the access of all kind of NCK observatory data.

Edited by: Jean Rasson

Reviewed by: Hans-Ulrich Auster and Alexandre Gonsette

\section{References}

Ádám, A., Bencze, P., Bór, J., Heilig, B., Kis, Á., Koppán, A., Kovács, K., Lemperger, I., Märcz, F., Martini, D., Novák, A., Sátori, G., Szalai, S., Szarka, L., Verő, J., Wesztergom, V., and Zieger, B.: Geoelectromagnetism and the changing Earth, Acta Geod. Geophys. Hu., 44, 271-312, https://doi.org/10.1556/AGeod.44.2009.3.3, 2009.

Brunke, H.-P. and Matzka, J.: Numerical Evaluation of magnetic absolute Measurements with arbitrary distributed DI-Fluxgate Theodolite Positions, Geosci. Instrum. Method. Data Syst. Discuss., https://doi.org/10.5194/gi-2017-3, in review, 2017.

Clarke, E., Baillie, O., Reay, J. S., and Turbitt, C. W.: A method for the near real-time production of quasi-definitive magnetic observatory data, Earth Planets Space, 65, 1363-1374, https://doi.org/10.5047/eps.2013.10.001, 2013.

Gilbert, D. and Rasson, J. L.: Effect on DIflux Measuring Accuracy due to a Magnet located on it, Scientific Technical Report STR98/21, GeoForschungsZentrum Potsdam, 1998. 
ISO 17123-3:2001: Optics and optical instruments - Field procedures for testing geodetic and surveying instruments - Part 3: Theodolites, Standard, International Organization for Standardization, Geneva, Switzerland, available at: https: //www.iso.org/obp/ui/\#iso:std:iso:17123:-3:ed-1:v1:en (last access: 19 July 2017), 2001.

Jankowski, J. and Sucksdorff, C.: IAGA Guide for Magnetic Measurements and Observatory Practice, International Association of Geomagnetism and Aeronomy, available at: http://www.iaga-aiga.org/data/uploads/pdf/guides/ iaga-guide-observatories.pdf (last access: 19 July 2017), 1996.

Newitt, L. R., Barton, C. E., and Bitterly, J.: Guide for Magnetic Repeat Station, International Association of Geomagnetism and Aeronomy, available at: http://ftp.bcmt.fr/pdf/ IAGA-Guide-Repeat-Stations.pdf (last access: 19 July 2017), 1996.
St-Louis, B. J., Sauter, E. A., and Coles, R. L.: InterMagnet Technical Reference Manual, version 4.6, InterMagnet, available at: http://www.intermagnet.org/publications/intermag_4-6.pdf (last access: 19 July 2017), 2012.

Vértessy, G., Gasparics, A., and Szöllősy, J.: High sensitivity magnetic field sensor, Sensor. Actuat. A-Phys., 85, 202-208, https://doi.org/10.1016/S0924-4247(00)00368-X, 2000.

Zhang, S., Fu, C., He, Y., Yang, D., Li, Q., Zhao, X., and Wang, J.: Quality Control of Observation Data by the Geomagnetic Network of China, Data Science Journal, 15, p. 15, https://doi.org/10.5334/dsj-2016-015, 2016. 